\title{
Mehr als immer nur dagegen? Die legislative Agenda grüner Parteien in Deutschland und Österreich in Zeiten Großer Koalitionen
}

\author{
Jasmin Fitzpatrick und Sabrina J. Mayer
}

Im Parlamentarischen Regierungssystem liegt bekanntlich die gesetzgeberische Initiative hauptsächlich in den Händen der Regierung im Zusammenspiel mit den sie tragenden (Mehrheits-)fraktionen. Jedoch ist das Initiativrecht kein Privileg der Regierungsmehrheit, sondern kann auch von der Opposition in unterschiedlicher rechtlicher Ausgestaltung, zum Beispiel als Antragsrecht einzelner Abgeordneter oder von Fraktionen, genutzt werden. Damit eröffnet sich taktischer Spielraum für Oppositionsfraktionen: Sie können versuchen, die politische Agenda mitzubestimmen, indem sie darauf setzen, dass die Regierung ihre Position in eigenen Gesetzen übernimmt, die Regierungsfraktionen in grundlegenden Fragen dazu zwingen, sich zu positionieren und/oder der Öffentlichkeit zeigen, für welche Themen sich die Partei in konkreter Weise einsetzt. Dieser letzte Aspekt ist besonders für Parteien der Opposition relevant, die unter Zwang zur Profilierung stehen, wenn die Regierungsmehrheit zentrale Themen der Opposition umgesetzt hat oder wenn das eigene Profil durch das Aufkommen neuer Kontrahenten oder politischer Streitfragen unscharf geworden ist.

Diesen Herausforderungen sahen sich besonders Bündnis 90/Die Grünen in der Bundesrepublik Deutschland ausgesetzt. Nach dem Wegfall des Kernthemas, dem Ausstieg aus der zivilen Atomkraftnutzung 2011, wurden weitere Themen wie Nachhaltigkeit, Ausbau direktdemokratischer Beteiligungsverfahren und Gleichheit aller Geschlechter auch vermehrt durch andere politische Parteien aufgegriffen. Dabei waren Forderungen nicht unbedingt deckungsgleich (beispielsweise in puncto Klimapolitik im Kontrast zu CDU/CSU und SPD), teilweise entstehen auch Konflikte hinsichtlich der Deutungshoheit (beispielsweise in der Forderung nach einem Ausbau direktdemokratischer Verfahren durch die AfD), doch im Ergebnis sind die Grünen nicht mehr die einzigen, die diese Politikfelder mit Inhalt füllen. Zudem wurden sie durch die stimmenstärkste Kontrahentin, die Union, lange nicht als Koalitionspartner auf Bundesebene in Betracht gezogen, und Mehrheiten im Mitte-Links-Lager waren unrealistisch. Dies lag auch an den Koalitionspräferenzen der Parteien, während seitens der eigenen Anhängerschaft ein rot-rot-grünes Regierungsbündnis durchaus als Option gesehen wurde. ${ }^{1}$ Das Aufkommen der Piraten, die zwischen 2009 und 2014 im jungen Wählerspektrum Fuß fassen konnten ${ }^{2}$, stellte eine weitere Herausforderung für die Grünen dar. ${ }^{3}$

Um zu analysieren, welche Konsequenzen sie aus diesen Herausforderungen für ihre programmatische Ausrichtung gezogen haben, sind verschiedene Herangehensweisen vorstellbar - etwa die Untersuchung von Wahlprogrammen im Rahmen einer Policyanalyse,

1 Vgl. Marc Debus, Koalitionspräferenzen von Wählern und Parteien und ihr Einfluss auf die Wahlabsicht bei der Bundestagswahl 2009, in: ZParl, 43. Jg. (2012), H. 1, S. $86-102$.

2 Vgl. Christoph Bieber, Piratenpartei Deutschland (PIRATEN), in: Frank Decker / Viola Neu (Hrsg.), Handbuch der deutschen Parteien, Wiesbaden 2018, S. 443 - 448, S. 444.

3 Vgl. Lothar Probst, Bündnis 90/Die Grünen (GRÜNE), in: Frank Decker / Viola Neu (Hrsg.), a.a.O. (Fn. 2), S. 203 - 218, S. 206. 
wie sie bereits von Reimut Zohlnhöfer ${ }^{4}$ durchgeführt wurde. Seine Studie führte zum Ergebnis, dass die Opposition durchaus die Positionen der Regierungsmehrheit verändern kann, vor allem durch das Angebot einer programmatischen Alternative bei öffentlich wahrgenommener Kompetenz im selben Policyfeld. Als Beispiel führt er unter anderem den deutschen Atomausstieg an. Der politische Wettbewerb lässt sich jedoch nicht nur durch Wahlprogramme, sondern auch über andere Instrumente untersuchen, die eine detailliertere Analyse ermöglichen.

In diesem Beitrag liegt daher der Fokus auf Gesetzesinitiativen, da diese bereits ausformuliert sind, vielfach Abschätzungen zu Folgen enthalten und dem Parlament relativ unmittelbar zur Diskussion und Abstimmung vorgelegt werden. ${ }^{5}$ Erst indem die Aufmerksamkeit auf bestimmte Themen gelenkt wird, wird die notwendige Grundlage für intraparlamentarisches und mediales Agenda-Setting geschaffen. Daher wird im Folgenden untersucht, für welche Themen die Grünen in Zeiten Großer Koalitionen das Initiativrecht nutzten und inwiefern sich ihre legislative Agenda im Zeitverlauf geändert hat. Hierfür wurde eine inhaltsanalytische Auswertung aller Gesetzentwürfe grüner Parteien in Deutschland und Österreich zwischen 2007 und 2008 sowie zwischen 2013 und 2017 durchgeführt. Neben den deutschen Grünen wird die grüne Partei Österreichs als Referenz einbezogen. Dies ermöglicht die Bewertung, ob eventuelle Veränderungen im parlamentarischen Auftreten auf den nationalen Kontext zurückzuführen sind oder ob sich Muster in beiden Ländern beobachten lassen.

\section{Zur Rolle von Oppositionsfraktionen im Deutschen Bundestag und im Österreichischen Nationalrat}

In Deutschland und Österreich haben Oppositionsfraktionen neben Kontrollrechten wie etwa dem Interpellationsrecht, der Möglichkeit, das Verfassungsgericht anzurufen (Fraktionen können nach Art. 93 Abs. 1 Nr. 1 GG ein Organstreitverfahren vor dem BVerfG erwirken; für eine abstrakte Normenkontrolle sind gemäß Art. 93 Abs. 1 Nr. 2 und 2a GG hingegen ein Viertel der Mitglieder des Bundestages nötig) oder Untersuchungsausschüsse einzurichten (hierzu sind gemäß Art. 44 GG ein Viertel der Mitglieder des Bundestages notwendig), auch die Option, potenziell gestalterisch an der Parlamentsarbeit teilzunehmen, indem sie gemäß $\$ 76$ GO-BT bzw. $\$ 26$ GOG-NR eigene Gesetzesvorschläge ${ }^{6}$ einbringen. ${ }^{7}$

In Zeiten sogenannter Großer Koalitionen, in denen die beiden größten Fraktionen lagerübergreifend miteinander die Regierung stellen, gestaltet sich die Oppositionsarbeit

4 Vgl. Reimut Zohlnhöfer, Zum Einfluss des Parteienwettbewerbs auf politische Entscheidungen, in: Sebastian Bukow / Uwe Jun (Hrsg.), Parteien unter Wettbewerbsdruck, Wiesbaden 2017, S. $15-37$.

5 Vgl. ebenda, S. 19.

6 Für die gleichen Sachverhalte werden in beiden Ländern unterschiedliche Begriffe genutzt. Wir greifen in dieser Arbeit einheitlich auf den Begriff Gesetzentwürfe zurück, wenn wir durch die Opposition eingebrachte konkrete Gesetzvorlagen oder in Österreich selbständige Initiativ- und Entschließungsanträge meinen.

7 Vgl. für Österreich: Wolfgang C. Müller, Austria: Imperfect Parliamentarism but Fully-Fledged Party Democracy, in: Kaare Strom / ders. / Torbjörn Bergmann (Hrsg.), Delegation and Accountability in Parliamentary Democracies, Oxford 2003, S. 221 - 252, S. 235 f. Vgl. für Deutschland: Thomas Saalfeld, Germany: Multiple Veto Points, Informal Coordination, and Problems of Hidden Action, in: ebenda, S. 347 - 375, S. 361 f. 
schwieriger. Dies galt im besonderen Maße für den deutschen Fall zwischen 2013 und 2017, als die Existenz einer „qualifiziert großen Koalition“8 verfassungsrechtliche Kontrollmöglichkeiten der Opposition einschränkte, weil diese die notwendigen Quoren nicht erreichte. In Österreich hingegen stellte diese Konstellation den „Normalfall“ dar. ${ }^{9}$ Im Nationalrat, der verfassungsrechtlich stärkeren Kammer, waren Bündnisse der beiden großen Parteien SPÖ und ÖVP nach 1945 mehr die Regel als die Ausnahme. Dieser Sachverhalt gewinnt besondere Brisanz, wenn man bedenkt, dass Verfassungsänderungen von „zwei Dritteln bei Anwesenheit der Hälfte der Abgeordneten "10 möglich sind.

In beiden betrachteten Parlamenten begann durch den Einzug der Grünen (in Deutschland 1983, in Österreich 1986) eine zunehmende Fragmentierung, die sich vor allem während der 2000er Jahre fortsetzte. ${ }^{11}$ Das Aufkommen dieser neuen politischen Kraft hatte jedoch in den beiden Parlamenten unterschiedliche Konsequenzen, die mit dem jeweiligen Arbeitsmodell zusammenhängen: Während im Nationalrat dem Plenum eine zentrale Rolle zukommt $^{12}$ („Tribünefunktion“13), haben im Bundestag hingegen die Ausschüsse im Zusammenwirken mit den Fraktionen eine zentrale Position in der Gesetzgebung. ${ }^{14}$ In Zeiten Großer Koalitionen werden die Ausschüsse des Nationalrats durch Regierungsfraktionen dominiert, so dass sichtbare Oppositionsarbeit nur im Plenum möglich ist. Dies führt dazu, dass die Opposition vermehrt Entschließungsanträge einbringt. ${ }^{15}$ Als Anzeichen der eher schwachen Stellung der Ausschüsse kann der Umstand gesehen werden, dass nur ein Ausschuss für ein Gesetz zuständig ist, während im Bundestag mehrere Ausschüsse am selben Entwurf arbeiten; in einem Ausschuss haben die Anträge der Opposition im Nationalrat eher geringe Erfolgsaussichten, auch nur beraten zu werden. ${ }^{16}$ Im Bundestag sind die Ausschüsse laut Geschäftsordnung vorbereitende Beschlussorgane, die teilweise sogar verfassungsrechtlich verankert sind (Art. 45, 45a und 45 b GG). Die Verteilung der Ausschussvorsitze wird in der Regel entsprechend $\$ 12$ GO-BT gemäß der Stärke der Fraktionen bestimmt, so dass auch Oppositionsfraktionen in einigen Ausschüssen den Vorsitz stellen. ${ }^{17}$ Die wohl nachhaltigsten Änderungen im österreichischen Fall sind der Ausbau der parlamentarischen Minderheits-

8 Vgl. Pascale Cancik, „Effektive Opposition“ im Parlament - eine ausgefallene Debatte?, in: ZParl, 48. Jg. (2017), H. 3, S. $516-534$.

9 Vgl. Christoph Konrath, Parlamentarische Opposition in Österreich: Recht und Praxis in Zeiten eines fragmentierten Parteiensystems in: ZParl, 48. Jg. (2017), H. 3, S. 557 - 574, S. 557.

10 Für Österreich: Art. 44 (2) B-VG; für Deutschland: Art. 79 (2) GG.

11 Vgl. für Österreich: Wolfgang C. Müller, a.a.O. (Fn. 7), S. $224 \mathrm{f}$. Vgl. für Deutschland: Thomas Saalfeld, a.a.O. (Fn. 7), S. 349 f.

12 Vgl. Christoph Konrath, a.a.O. (Fn. 9), S. 563.

13 Hubert Sickinger, Die Funktion der Nationalratsausschüsse im Prozess der Gesetzgebung, in: Österreichische Zeitschrift für Politikwissenschaft, 29. Jg. (2000), H. 2, S. 157 - 176, S. 164.

14 Vgl. Sven T. Siefken, Plenum im Kleinen oder Ort der Verhandlung? Verständnisse und Forschungsbedarf zu den Fachausschüssen des Deutschen Bundestages, in: ZParl, 49. Jg. (2018), H. 4, S. 777 - 792; Jürgen von Oertzen, Das Expertenparlament. Abgeordnetenrollen in den Fachstrukturen bundesdeutscher Parlamente, Baden-Baden 2006; Suzanne S. Schüttemeyer, Fraktionen im Deutschen Bundestag 1949-1997. Empirische Befunde und theoretische Folgerungen, Opladen 1998.

15 Vgl. Christoph Konrath, a.a.O. (Fn. 9).

16 Vgl. Hubert Sickinger, a.a.O. (Fn. 13), S. 164.

17 Vgl. für ausführlichere Darstellung: Wolfgang Ismayr, Der Deutsche Bundestag, Wiesbaden 2012, S. $166 \mathrm{ff}$. 
rechte nach den Geschäftsordnungsreformen $1996^{18}$ bzw. ab 2008, die notwendig geworden waren, um die Zustimmung der Grünen zu wichtigen Gesetzen in EU-Angelegenheiten zu erhalten. Die österreichischen Grünen konnten ihr „Erpressungspotenzial“19 wortwörtlich geltend machen und den regierenden Fraktionen Zugeständnisse abringen, die sich an der Stellung der Opposition im Deutschen Bundestag orientierten. ${ }^{20}$

Hinsichtlich der Organisation der Plenarsitzungen finden sich auch Gemeinsamkeiten: Fraktionen (Bundestag) und Klubs (Nationalrat) sind bei der Rednerauswahl, bei Abstimmungen und bei der inhaltlichen Arbeit maßgeblich. Hubert Sickinger betont die Rolle der Klubs/Fraktionen: In der „Praxis sind Abgeordnete de facto nur über ihre Fraktionen (Klubs) politisch handlungsfähig“21. Im Gesetzgebungsprozess des Bundestages können Fraktionen (oder fünf Prozent der Abgeordneten) das Initiativrecht wahrnehmen und Gesetzesvorschläge einbringen (\$ 76 (1) GO-BT). Im Nationalrat können fünf Abgeordnete gemeinsam einen selbständigen Antrag einbringen oder einen Entschließungsantrag fordern (Resolutionsrecht), der lediglich Eckpunkte eines Gesetzes liefert und die Regierung dazu auffordert, einen Gesetzentwurf einzubringen. Meistens gingen beschlossene Gesetze jedoch auf eine Regierungsvorlage zurück. ${ }^{22}$ Die Erfolgsaussichten von Gesetzesinitiativen sind auch im Bundestag gering: So waren im Zeitraum von 1990 bis 2017 nur vier der 972 Gesetzentwürfe, die ausschließlich auf Oppositionsfraktionen zurückgingen, erfolgreich. ${ }^{23}$

Der Bundesrat bietet im deutschen System für die Oppositionsfraktionen Gestaltungsmöglichkeiten, da auch dieses Verfassungsorgan ein Gesetzesinitiativrecht hat (Art. 76 (1) GG). Jedoch ist vor einer Befassung des Bundestages eine Stellungnahme der Bundesregierung notwendig, bevor der Gesetzesvorschlag den legislativen Weg durch den Bundestag nimmt. Im Bundesrat wird zudem seit den 1970 er Jahren eine Parteipolitisierung ${ }^{24}$ beobachtet, die Optionen für die Oppositionsfraktionen des Bundestages ermöglicht, sofern sie an Regierungen in mehreren Bundesländern beteiligt sind. Da es in diesem Beitrag jedoch um die Möglichkeiten der Oppositionsfraktionen im Bundestag geht, wird hierauf nicht weiter eingegangen.

\section{Funktionen chancenloser Oppositionsinitiativen}

Auch ohne erfolgreiche Verabschiedung kann ein oppositioneller Gesetzentwurf in zweierlei Hinsicht einen Zweck erfüllen: Er kann als Mittel genutzt werden, um die Regierung dazu zu zwingen, sich zu positionieren und gleichzeitig eigene inhaltliche Punkte öffentlich

18 Vgl. Hubert Sickinger, a.a.O. (Fn. 13), S. 165.

19 Vgl. Giovanni Sartori, Parties and Party Systems: A Framework for Analysis, Cambridge 1976.

20 Vgl. Christoph Konrath, a.a.O. (Fn. 9), S. 566.

21 Vgl. Hubert Sickinger, a.a.O. (Fn. 13), S. 159.

22 Vgl. ebenda, S. 162. Genaue Zahlen sind in den Bilanzberichten des Nationalrats leider nicht ausgewiesen und können daher nicht berichtet werden.

23 Vgl. Datenhandbuch zur Geschichte des Deutschen Bundestages, Kapitel 10.1 Statistik zur Gesetzgebung, Stand: 5. März 2018, https://www.bundestag.de/resource/blob/196202/ ee30d500ea94ebf8146d0ed7b12a8972/Kapitel_10_01_Statistik_zur_Gesetzgebung-data.pdf (Abruf am 22. März 2021).

24 Vgl. Gerhard Jahn / Roman Herzog, Fehlentwicklungen im Verhältnis von Bundesrat und Bundestag?, in: ZParl, 7. Jg. (1976), H. 3, S. 291 - 316; Isabella Harle / Christian Stecker, Die Initiativtätigkeit des Bundesrates im Lichte der Parteipolitisierungsthese, in: ZParl, 42. Jg. (2011), H. 2, S. $325-334$. 
zu platzieren - also die Agenda zu besetzen. Mit Agenda-Setting wird dabei eine Spezifizierung dessen vorgenommen, was in der Trias der Oppositionsfunktionen ${ }^{25}$ neben Kritik und Kontrolle als das Aufzeigen von Alternativen verstanden wird. Hier soll somit keine zusätzliche Funktion vorgeschlagen werden, sondern eine Konkretisierung durch die Verbindung mit dem in der politischen Kommunikationsforschung verbreiteten Ansatz des AgendaSettings erreicht werden. Wenn von Agenda-Setting die Rede ist, wird häufig unweigerlich an den Begriff von Maxwell E. McCombs und Donald L. Shaw gedacht. ${ }^{26}$ Dieser bezieht sich jedoch eher auf die massenmediale Agenda und fragt, was die Menschen wahrnehmen und wie dies ihr politisches Verhalten beeinflusst. Agenda-Setting kann auch in anderen Kontexten untersucht werden, wie etwa dem Parlament. Hier kann es als Instrument dafür gesehen werden, inhaltliche Alternativen sichtbar zu machen. Bei der Untersuchung von innerparlamentarischem Agenda-Setting steht beispielsweise die Wechselwirkung zwischen dem parlamentarischen Handeln verschiedener Fraktionen im Fokus. ${ }^{27}$ Diese wissenschaftliche Auseinandersetzung hat eine gewisse Tradition. So führte bereits Giovanni Sartori aus, dass Parteien aufgrund von zwei Aspekten Bedeutung erlangen können: aufgrund ihres Koalitions- oder Erpressungspotenzials. Letzteres bezieht sich auf oppositionelles Handeln von Antisystemparteien. ${ }^{28}$ Jedoch können auch systemtreue Oppositionsfraktionen Druck aufbauen, indem sie inhaltliche Alternativen aufzeigen, die in der öffentlichen Meinung Zuspruch finden.

Obwohl diese Zusammenhänge wissenschaftliche Aufmerksamkeit verdienen, wird Oppositionshandeln in der Parlamentarismusforschung bis jetzt allerdings nur wenig beachtet. ${ }^{29}$ Noch seltener gibt es Studien, die eine vergleichende Perspektive einnehmen. Jedoch lassen sich in der Literatur einige Fallstudien zu einzelnen Ländern finden, die sich der Betrachtung von Oppositionshandeln widmen. 1992 analysierte Martin Sebaldt den Ursprung der Gesetze im Deutschen Bundestag mit Fokus auf die oppositionelle Initiativtätigkeit. Ein zentrales Ergebnis besteht darin, „daß das Thematisierungspotential der Opposition nicht ernsthaft in Abrede gestellt werden kann" ${ }^{30}$. Neuere Studien zum deutschen Fall befassen sich auch unterhalb der Bundesebene mit Agenda-Setting durch die Opposition. Zu nennen wäre hier etwa die umfassende Studie von Karina Hohl zum Nordrhein-Westfälischen Landtag. ${ }^{31}$ Katrin Praprotnik betrachtete in ihrer Analyse zur Anwend-

25 Vgl. Marcus Höreth / Jörn Ketelhut, Was ist effektive Opposition? Überlegungen zu einem Schlüsselbegriff der Regierungslehre, in: Stephan Bröchler / Manuela Glaab / Helmar Schöne (Hrsg.), Kritik, Kontrolle, Alternative. Regierungssystem und Regieren in der Bundesrepublik Deutschland, Wiesbaden 2020, S. 95 - 118.

26 Vgl. Maxwell E. McCombs / Donald L. Shaw, The Agenda-Setting Function of the Mass Media, in: The Public Opinion Quarterly, 36. Jg. (1972), H. 2, S. 176 - 187.

27 Vgl. Rens Vliegenthart / Stefaan Walgrave / Corine S. Meppelink, Inter-Party Agenda-Setting in the Belgian Parliament: The Role of Party Characteristics and Competition, in: Political Studies, 59. Jg. (2011), S. $368-387$, S. 368.

28 Vgl. Giovanni Sartori, a.a.O. (Fn. 19), S. 123 f.

29 Vgl. Henrik Bech Seeberg, Opposition Policy Influence through Agenda-Setting: The Environment in Denmark, 1993-2009, in: Scandinavian Political Studies, 39. Jg. (2016), H. 2, S. 185 206, S. 187.

30 Martin Sebaldt, Innovation durch Opposition: Das Beispiel des Deutschen Bundestages 19491987, in: ZParl, 23. Jg. (1992), H. 2, S. 238 - 265, S. 259.

31 Vgl. Karina Hohl, Agenda Politics im Parlament. Das Themen- und Tagesordnungsmanagement der Opposition im Landtag von NRW, Wiesbaden 2018. 
barkeit der Mandatstheorie sowohl Regierungs- als auch Oppositionsfraktionen in Österreich. Anhand eines Abgleichs der in den Wahlprogrammen gegebenen Wahlversprechen und der tatsächlich zustande gekommenen Gesetze fand sie heraus, dass die Oppositionsfraktionen im von ihr betrachteten Zeitraum Wahlversprechen nur dann umsetzen konnten, wenn „diese auch Teil der Programme der Regierungsfraktionen waren “32. Wann diese Schnittmengengesetze verabschiedet worden sind und ob hier taktisch im Kontext bevorstehender Wahlen gehandelt worden ist, lässt sich aus dieser Studie nicht ablesen. Für den belgischen Fall wurde untersucht, wie sich Abgeordnete gegenseitig beeinflussen und somit Agenda-Setting-Potenzial entsteht. ${ }^{33}$ Diese Analyse zählt zu einer Reihe von Studien, die sich mit innerparlamentarischem Agenda-Setting auseinandersetzen. In ähnlicher Weise wurde für Dänemark gezeigt, dass Oppositionsfraktionen, die ,issue owner" sind, also die Hoheit über ein bestimmtes Thema beanspruchen, auf diesem Feld die Regierungsmehrheit dazu bewegen können, Gesetze gegen ihre erwartete ideologische Position zu verabschieden und beispielsweise rechte oder konservative Fraktionen eher linksorientierte Umweltpolitik verfolgen. ${ }^{34}$

Etwas Ähnliches war im deutschen Fall nach 2011 zu beobachten. Hier vollzog eine konservative Regierung, die sich erst ein halbes Jahr zuvor klar zur nuklearen Energiegewinnung bekannt hatte, nach dem Unglück in Fukushima die atomare Kehrtwende in beispielloser Eile. Zwar gab es bereits bis 2010 Pläne für einen Atomausstieg, die auch unter der Großen Koalition zwischen 2005 und 2009 nicht rückgängig gemacht wurden. Die CDU und ihr präferierter Juniorpartner, die FDP, äußerten sich damals jedoch kritisch, so dass das Thema ein Streitpunkt blieb. Der jahrelange politische Druck durch die Grünen, der Atomunfall in Japan und die öffentliche Skepsis gegenüber der nuklearen Energiegewinnung schufen ein Szenario, das den damaligen Regierungsfraktionen CDU und FDP ein Fiasko bei den bevorstehenden Landtagswahlen in Baden-Württemberg, Rheinland-Pfalz und Sachsen-Anhalt prognostizierte. Während der Druck und der Einfluss der Oppositionspartei auf die Regierungsfraktionen hier also deutlich werden, bleibt ungeklärt, wie die grüne Opposition, deren Markenkern plötzlich eine „politische Kernschmelze“ erlitt, reagierte. Diese Gegenreaktion auf Themenübernahmen seitens betroffener Oppositionsfraktionen findet insgesamt in der Forschung keine Berücksichtigung.

Während das Jahr 2011 für die parteipolitische Auseinandersetzung in Deutschland große Veränderungen brachte, blieben diese im Vergleichsland Österreich aus. Das lag vor allem daran, dass dort nukleare Energiegewinnung keine politische Streitfrage ist. Seit 1999 ist in Österreich der Bau von Anlagen zur nuklearen Energiegewinnung sogar verfassungsrechtlich verboten - und solche Anlagen hat es in Österreich auch zuvor nie gegeben. ${ }^{35}$ Ein Vergleich der grünen Parteien beider Länder ist also sinnvoll. Wenn eine Veränderung der thematischen Ausrichtung in beiden Betrachtungsfällen parallel zu beobachten ist, könnte dies auf andere, noch zu identifizierende Faktoren zurückzuführen sein. Außerdem ist denkbar, dass es bei den deutschen Grünen nach dem fast vollkommenen Wegfall des

32 Katrin Praprotnik, Die Policy-Bilanz der Regierung Faymann I. Ein Test der Mandatstheorie, in: Österreichische Zeitschrift für Politikwissenschaft, 44. Jg. (2015), H. 2, S. 1 - 14, S. 8.

33 Vgl. Rens Vliegenthart / Stefaan Walgrave / Corine S. Meppelink, a.a.O. (Fn. 27).

34 Vgl. Henrik Bech Seeberg, a.a.O. (Fn. 29).

35 Vgl. ENU - Energie- und Umweltagentur des Landes Niederösterreich, Atomkraft in Österreich, https://www.enu.at/atomkraft-in-oesterreich-einleitung (Abruf am 25. September 2019). 
Atomausstiegsthemas zu einer weiteren inhaltlichen Annäherung an den europäischen Nachbarn gekommen ist. Die österreichischen Grünen haben seit dem Grundsatzprogramm von 2001 als Kernthemen vor allem das Verhältnis von Wirtschaft und Umwelt, faire Lohnpolitik (Fairteilen) und eine offene solidarische Gesellschaft gesetzt. ${ }^{36}$ Diese Punkte waren zwar auch im Themenportfolio der deutschen Grünen bereits enthalten, jedoch bestand im klaren Nein zur nuklearen Energiegewinnung ein deutlicher Unterschied zu den Regierungsfraktionen (vor allem der CDU) stieß das Thema in der Bevölkerung auf große Unterstützung. ${ }^{37}$ Auf anderen Politikfeldern wurde den Grünen laut Umfragen wenig Lösungskompetenz zugesprochen. ${ }^{38} \mathrm{Um}$ sich zu profilieren, musste die Partei also „issue ownership“ für neue Themen glaubhaft beanspruchen. Solche Fokusverschiebungen sollten auch im Parlamentshandeln sichtbar werden.

\section{Gesetzesinitiativen als Untersuchungsgrundlage: Daten und Operationalisierungen}

Um den Einfluss externer Ereignisse halbwegs konstant zu halten, sollten die Untersuchungszeiträume in beiden Ländern identisch sein. Da in Deutschland Große Koalitionen weitaus seltener sind/waren als in Österreich und ein aktueller Zeitraum betrachtet werden sollte, wurde als erster Untersuchungszeitraum die 16. Wahlperiode in Deutschland, und zwar die Jahre 2005 bis 2008 ausgewählt. Mit der Regierung Alfred Gusenbauer bestand allerdings erst ab Januar 2007 zeitgleich eine Große Koalition in Österreich, die nach der Nationalratswahl im September 2008 noch bis Dezember 2008 im Amt war (XXIII. Gesetzgebungsperiode). Hierbei ähneln sich die Voraussetzungen, da auch in Österreich erstmalig nach einiger Zeit (wenn auch nur sieben Jahre im Vergleich zu den 36 Jahren in Deutschland) wieder eine Große Koalition regierte. Die dritte Große Koalition in Deutschland bestand während der 18. Wahlperiode von Dezember 2013 bis zur Bundestagswahl im September 2017 und verblieb geschäftsführend noch bis März 2018 im Amt. Zur selben Zeit bestand nach der Nationalratswahl 2013 auch in Österreich eine Große Koalition von Dezember 2013 bis Dezember 2017, die die Regierungen Werner Faymann II sowie Christian Kern umfasste (XXV. Gesetzgebungsperiode). Als Untersuchungszeitraum sind daher die Phasen Januar 2007 bis Dezember 2008 sowie Dezember $2013^{39}$ bis Dezember 2017 geeignet, in denen in beiden Ländern eine Große Koalition bestand. Für eine Kongruenz

36 Vgl. Die Grünen, Grundsatzprogramm der Grünen, https://web.archive.org/web/2012013 1045358/http://ooe.gruene.at/fileadmin/oberoesterreich/benutzerinnen/downloads/gruene_ bund_grundsatzprogramm2001.pdf (Abruf am 25. September 2019).

$37 \mathrm{Vgl}$. Zeit online, Zehntausende protestieren gegen Atomkraft, 18. September 2010, https://www. zeit.de/politik/deutschland/2010-09/demonstration-atomenergie-berlin (Abruf am 25. September 2019).

38 Vgl. für die Bundestagswahl 2009: Ossip Fürnberg / Markus Steinbrecher, Wen kümmern schon Inhalte? Die Bedeutung von Themenorientierungen für das Wahlverhalten in ausgewählten Bevölkerungsgruppen bei der Bundestagswahl 2009, in: Bernhard Weßels / Harald Schoen / Oscar W. Gabriel (Hrsg.), Wahlen und Wähler, Wiesbaden 2013, S. 151 - 173. Vgl. für die Landtagswahlen 2011 in Baden-Württemberg: Forschungsgruppe Wahlen, Landtagswahl in Baden-Württemberg 2011. Kurzanalyse, https://www.forschungsgruppe.de/Wahlen/Wahlanalysen/Newsl_ Bade11.pdf (Abruf am 6. Dezember 2019).

39 Im Dezember 2013 wurden nur in Deutschland zwei Initiativen eingebracht, die jedoch nicht exkludiert werden sollten. 
der Zeiträume wurde der zweite ebenfalls in Zwei-Jahres-Abschnitte aufgeteilt: Dezember 2013 bis Dezember 2015 und Januar 2016 bis Dezember 2017.

Als Datengrundlage dienen alle Gesetzesinitiativen - Gesetzentwürfe in Deutschland, selbständige Anträge in Österreich - unter Beteiligung von Politikerinnen und Politikern der Grünen in den beiden Untersuchungszeiträumen. ${ }^{40}$ Bei jedem Gesetzentwurf wurde erfasst, ob er gemeinsam mit anderen Fraktionen oder allein eingereicht wurde, bzw. ob es sich um ein Änderungsgesetz handelte oder um ein neues Gesetz. Für die Erfassung als Änderungsgesetz wurde der Drucksachentitel verwendet, und die Drucksachen mit dem Begriff „Änderung“ im Titel als 1 und alle anderen als 0 kodiert.

Für die inhaltsanalytische Einordnung der Gesetzesinitiativen diente die deutsche Variante des Comparative Agendas Project Master Codebooks ${ }^{41}$ in der Version 2.5 ${ }^{42}$. Das Codebuch besteht dabei aus 22 sogenannten Major Codes, die Politikfelder abdecken wie Energie, Wohnungswesen und Raumordnung oder Außenhandel. Zudem gibt es 224 Minor Codes, die einzelne Teilbereiche erfassen, beispielsweise für den Major Code Energie die Minor Codes Kernenergie und Atomkraftwerke oder Kohle. ${ }^{43}$ Zur Untersuchung der Frage, ob vor allem nach dem Atomausstieg der Fokus der Gesetzesinitiativen stark auf Themen gerichtet wurde, für die die Grünen Themenhoheit beanspruchen, wurde im Vorfeld definiert, was unter grünen Themen zu verstehen ist. Gemäß den Minor Codes gelten als grüne Kernthemen alle Gebiete, die in den aktuellen Programmen der Grünen behandelt werden. Hierdurch wird deutlich, welche Policy-Bereiche die Grünen für sich reklamieren und dadurch „issue ownership“ vorantreiben. ${ }^{44} 49$ der 224 Minor Codes wurden als grüne Themen identifiziert. ${ }^{45}$

40 Diese erhielten wir über die jeweiligen Dokumentationsdienste der Parlamente im Volltext/PDF. Unsere Datenquellen waren dabei „Parlament aktiv“ in Österreich mit der Suche nach selbständigen Anträgen der Grünen bzw. unter Beteiligung der Grünen sowie DIP (Dokumentations- und Informationssystem für Parlamentarische Vorgänge) in Deutschland mit erweiterter Drucksachensuche, Körperschaftliche Urheber: Fraktion B90/GR.

41 Vgl. Shaun Bevan, Gone Fishing: The Creation of the Comparative Agendas Project Master Codebook, in: Frank R. Baumgartner / Christian Breunig / Emiliano Grossman (Hrsg.), Comparative Policy Agendas: Theory, Tools, Data, Oxford 2019, S. 18 - 34.

42 Vgl. Christian Breunig / Tinette Schnatterer, German Policy Agendas - Data Set and Descriptive Insights, Working Paper 2018 - University of Konstanz.

43 Über das German Policy Agendas (GPA) Project erhielten wir auch die Kodierung der Gesetzesinitiativen der deutschen Grünen. Die Kodierung der anderen Gesetzesinitiativen wurde unabhängig voneinander von zwei studentischen Hilfskräften vorgenommen. Diese erhielten zur Übung dasselbe zufällig gezogene Sample von 15 Gesetzen aus der bereits erfolgten GPA-Kodierung. Sie sollten diese Gesetzentwürfe zuerst selbst kodieren sowie anschließend die eigene Kodierung mit der GPA-Kodierung vergleichen und letztere dabei als „Goldstandard“ behandeln. Für die Kodierung der österreichischen Entwürfe 2007 bis 2008 sowie der deutschen 2013 bis 2017 haben wir jeweils die Übereinstimmung zwischen den beiden Kodierern überprüft, die 76 Prozent (Österreich 2013 bis 2017) und 78 Prozent (Österreich 2007 bis 2008) betrug. Divergierende Fälle wurden von den Autorinnen geprüft und zugeordnet. Wir möchten hiermit herzlich Christian Breunig für die Übermittlung der Daten danken.

44 Hierzu wurden zunächst von beiden Autorinnen unabhängig Einschätzungen zu grünen Themen vorgenommen, die auf Beobachtungen der Medienberichterstattung basieren. Diese wurden anschließend abgeglichen.

45 Für alle Analysen haben wir die Kodierung in einen Stata-Datensatz überführt, der zusammen mit dem Do-file für Re-Analysen unter https://osf.io/us89x/ erhältlich ist. Eine Liste aller 49 Minor Codes ist online unter http://sabrinajmayer.de/fitzpatrick-mayer-2021-mehr-als-immernur-dagegen-online-anhang zu finden. 


\section{Die legislative Agenda grüner Parteien in Zeiten Großer Koalitionen}

In Deutschland wurden in der 16. (für die Jahre 2005 bis 2008) und 18. Wahlperiode 972 bzw. 788 Gesetzentwürfe eingebracht, 264 bzw. 148 davon durch den Bundestag. ${ }^{46}$ Von den Gesetzentwürfen der Grünen, die sie allein eingereicht hatten, wurde kein einziger erfolgreich beschieden, von den Entwürfen gemeinsam mit anderen Fraktionen zwölf bzw. fünf. Eine ähnliche Übersicht für Österreich ist leider nicht verfügbar. Christoph Konrath berichtet jedoch umfassend von der Behandlung oppositioneller Anträge in Ausschüssen und der Tendenz diese zu vertagen oder abzulehnen. ${ }^{47}$ Daher nehmen wir an, dass von den allein vorgelegten Entwürfen der Grünen kein einziger verabschiedet wurde. Es sollte somit allen beteiligten Akteuren klar sein, dass ein eingereichter Entwurf nicht zur erfolgreichen Verabschiedung führen wird. Daher ist plausibel anzunehmen, dass diese Gesetzentwürfe bewusst genutzt werden, um andere Funktionen zu erfüllen.

In Abbildung 1 ist die Verteilung der eingebrachten Gesetzesinitiativen nach Zeitraum abgetragen. Dabei zeigt sich besonders in Österreich die Tendenz, dass die Initiativtätigkeit zum Ende der Wahlperiode stark zunimmt, was darauf hindeuten könnte, dass diese Einreichungen Öffentlichkeit für die jeweiligen Themen herzustellen.

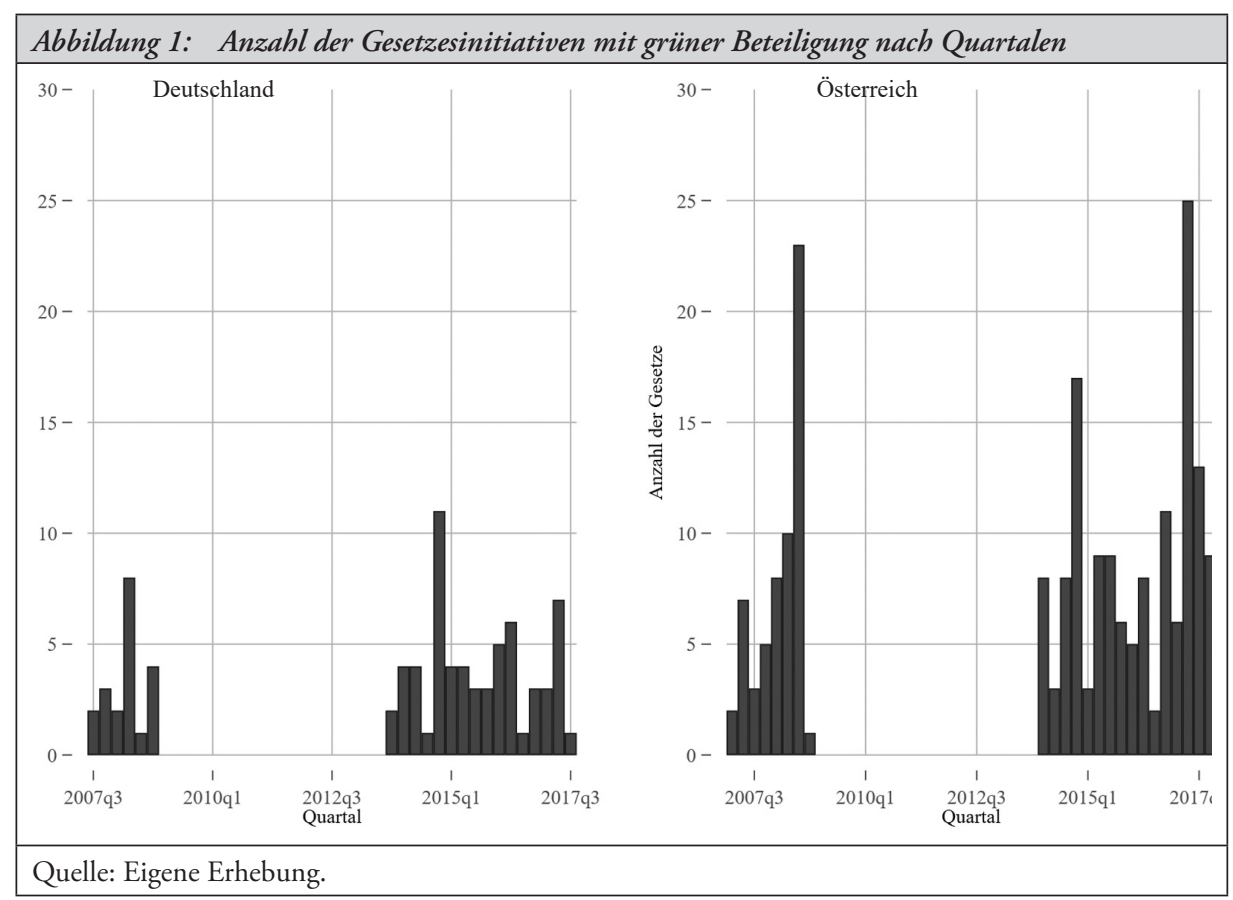

46 Vgl. Deutscher Bundestag, Statistik der Gesetzgebung der 16. und 18. Wahlperiode, https:// www.bundestag.de/parlamentsdokumentation (Abruf am 18. März 2021). Siehe auch Datenhandbuch zur Geschichte des Deutschen Bundestages, a.a.O. (Fn. 23).

47 Vgl. Christoph Konrath, a.a.O. (Fn. 9), S. 568 f. 
Aus den Zahlenangaben zu den Gesetzentwürfen mit Beteiligung der Gründen (siehe Tabelle 1) werden bereits zwei große Unterschiede sichtbar. Zum einen wird die Initiativtätigkeit in Österreich wesentlich stärker (fast doppelt bzw. mehr als doppelt so häufig) von den Grünen genutzt als in Deutschland. Zum anderen liegt der Anteil der Änderungsgesetze in Österreich deutlich über jenem in Deutschland. Es scheint, als ob die Grünen in Deutschland kreativer agieren, während die Grünen in Österreich eher reagierend Änderungen bestehender Gesetze fordern. Bei der Quote der Gesetze in alleiniger Einreichung haben sich die österreichischen und deutschen Grünen-Fraktionen mittlerweile angenähert. Während sie in Deutschland 2007 bis 2008 bei 75 Prozent lag, befindet sich der Anteil nun in beiden Ländern für den Zeitraum 2013 bis 2017 bei etwa 82 bis 84 Prozent. Diese Entwicklung zeigt, dass auch die deutschen Grünen mehr auf das Mittel des Gesetzentwurfs für die eigenständige Profilbildung zurückgreifen, die bei alleiniger Einreichung wesentlich ausgeprägter möglich ist.

\begin{tabular}{|c|ccc|ccc|}
\hline \multicolumn{1}{|c|}{ Tabelle 1: Übersicht über die zu kodierenden Gesetzentwürfe mit Beteiligung der Grünen } \\
\hline & \multicolumn{3}{|c|}{ Deutschland } & \multicolumn{3}{c|}{ Österreich } \\
& $2007-$ & $2013-$ & $2016-$ & $2007-$ & $2013-$ & $2016-$ \\
& 2008 & 2015 & 2017 & 2008 & 2015 & 2017 \\
\hline $\begin{array}{l}\text { Anzahl der Gesetzesinitiativen } \\
\text { davon allein (in Prozent) }\end{array}$ & 20 & 36 & 26 & 59 & 63 & 79 \\
$\begin{array}{l}\text { davon Änderungsgesetze } \\
\text { (in Prozent) }\end{array}$ & 75,0 & 86,1 & 80,8 & 89,8 & 85,7 & 78,5 \\
\hline Quelle: Eigene Erhebung. & 55,0 & 41,7 & 53,9 & 88,4 & 84,1 & 93,7 \\
\hline
\end{tabular}

Für eine erste inhaltliche Einordnung sind in Tabelle 2 die drei jeweils wichtigsten Major Codes ausgewiesen. Es ist leicht zu sehen, dass in das Feld der Bürger- und Freiheitsrechte sowie Minderheitsrechte die meisten Gesetzentwürfe fallen. Beispiele hierfür sind Diskriminierung aufgrund von Geschlecht und sexueller Ausrichtung ( $\mathrm{n}=14$ über alle vier Länder/Zeiträume, am häufigsten vier Gesetzentwürfe in Deutschland 2013 bis 2015 und Österreich 2007 bis 2008) sowie Einwanderung, Flüchtlinge, Aussiedler und Einbürgerung ( $\mathrm{n}=18$, acht Gesetzentwürfe in Deutschland 2013 bis 2015). Auch die Kategorie Öffentliche Verwaltung ist jeweils stark vertreten. Hier fallen die meisten Gesetzentwürfe im Minor Code „Parlamentarische und ministeriale Beziehungen“ an ( $\mathrm{n}=26$, neun in Österreich 2013 bis 2015). Dabei handelt es sich um Geschäftsordnungen, Bundesverfassungsgesetze, aber auch Änderungen an der Finanzierung der Fraktionen/Klubs oder dem Zuschnitt der Ministerien - alles Sachverhalte, die in jeder Wahl-/Gesetzgebungsperiode erneut anfallen und bei denen die Grünen oftmals mit anderen Fraktionen zusammenarbeiten, um gemeinsam die parlamentarische Organisation zu steuern: Zwölf der Entwürfe in dieser Kategorie sind dabei in der Tat gemeinsam mit anderen Fraktionen erfolgt. Betrachtet man daher nur die Gesetzentwürfe, die allein eingebracht wurden, dann ist dieser Code in Deutschland 2013 bis 2017 nicht mehr unter den drei wichtigsten und in Österreich zu keiner Zeit mehr auf dem ersten Platz. Im Bereich Recht, Kriminalität und Familienangelegenheiten gibt es keine eindeutigen Trends für die Minor Codes: Hier erstrecken sich die Gesetzesinitiativen über eine Vielzahl von Kategorien. 


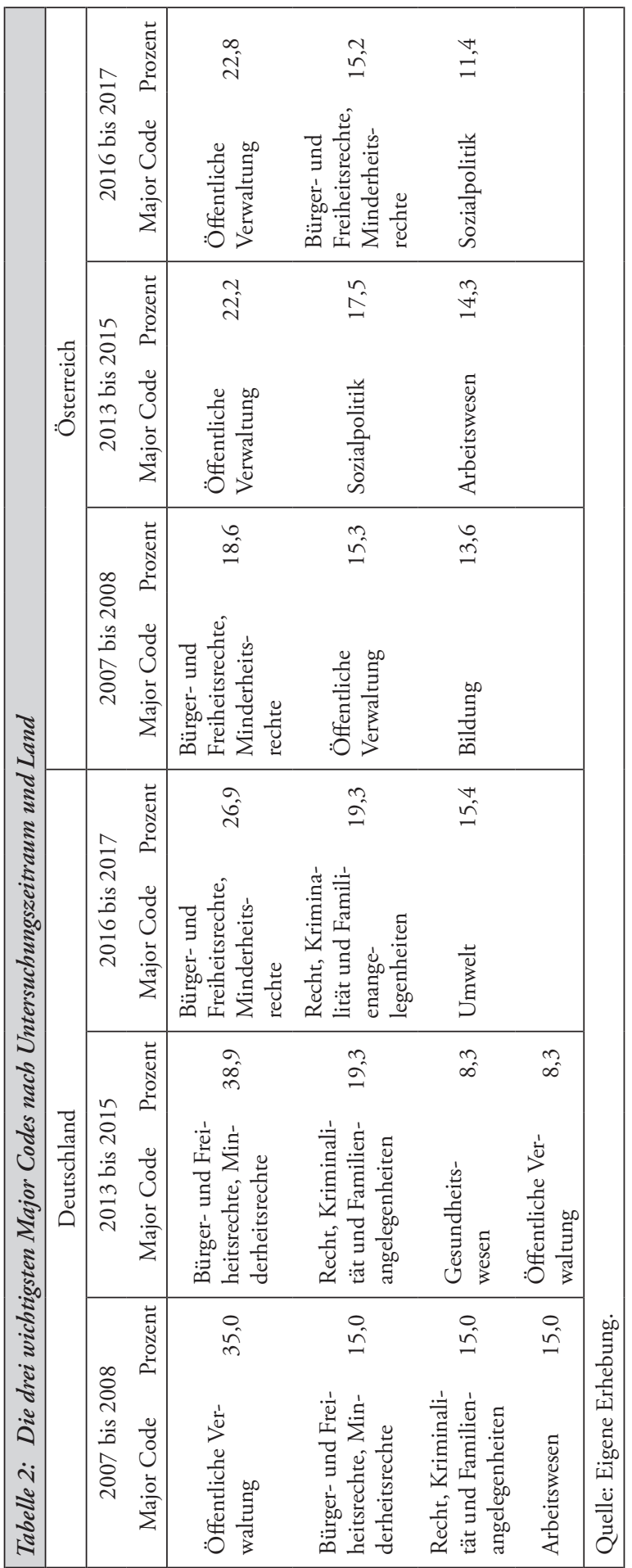




\begin{tabular}{|c|c|c|c|c|c|c|}
\hline & \multicolumn{3}{|c|}{ Deutschland } & \multicolumn{3}{|c|}{ Österreich } \\
\hline & $\begin{array}{c}2007- \\
2008 \\
\end{array}$ & $\begin{array}{c}2013- \\
2015 \\
\end{array}$ & $\begin{array}{c}2016- \\
2017 \\
\end{array}$ & $\begin{array}{c}2007- \\
2008 \\
\end{array}$ & $\begin{array}{r}2013- \\
2015 \\
\end{array}$ & $\begin{array}{c}2016- \\
2017 \\
\end{array}$ \\
\hline Grüne Kernthematik: Ja (in Prozent) & 20,0 & 71,0 & 42,9 & 45,3 & 31,5 & 35,5 \\
\hline $\begin{array}{l}\text { Anzahl der Minor Codes, in denen } \\
\text { die Gesetzentwürfe mit Grüner Kern- } \\
\text { thematik verortet werden }\end{array}$ & 3 & 11 & 5 & 14 & 11 & 26 \\
\hline
\end{tabular}

Für eine Untersuchung, in welchem Ausmaß grüne Themen forciert werden, liegt es nahe, nur die Gesetzentwürfe in alleiniger Urheberschaft zu berücksichtigen. So können zum einen prozedurale Vorlagen ausgeschlossen werden. Zum anderen kann angenommen werden, dass die Nutzung von Initiativen zur Setzung von Themen vor allem hier erfolgt, da bei diesen Entwürfen keine Abstimmung mit anderen Fraktionen notwendig ist (siehe Tabelle 3).

Vor 2011 ist kein Gesetzentwurf der Grünen im Bundestag für das Themenfeld Atomausstieg/Atomare Sicherheit zu finden. Ein möglicher Grund hierfür liegt im Koalitionsvertrag der zweiten Großen Koalition. Dort heißt es: „Zwischen CDU, CSU und SPD bestehen hinsichtlich der Nutzung der Kernenergie zur Stromerzeugung unterschiedliche Auffassungen. Deshalb kann die am 14. Juni 2000 zwischen Bundesregierung und Energieversorgungsunternehmen geschlossene Vereinbarung und können die darin enthaltenen Verfahren sowie für die dazu in der Novelle des Atomgesetzes getroffene Regelung nicht geändert werden. “48 Dieser Verweis gefährdet den Atomausstieg nicht unmittelbar, weshalb andere Sachfragen für Bündnis 90/Die Grünen Priorität gewonnen haben könnten. Dieses Thema war also bereits vor dem Atomausstieg nicht von Bedeutung für die Einbringung von chancenlosen Initiativen. Stattdessen wurden nach dem Ausstieg, vor allem von 2016 bis 2017, vier Entwürfe initiiert, von denen sich drei auf die Suche nach einem Endlager bezogen.

Im Untersuchungszeitraum nahmen in Deutschland die Gesetzentwürfe mit grüner Kernthematik um mehr als 51 (2007 bis 2008 zu 2013 bis 2015) bzw. 23 (2007 bis 2008 zu 2016 bis 2017) Prozentpunkte zu; mehr als die Hälfte aller Vorlagen bezogen sich 2013 bis 2017 auf grüne Kernthemen. Die Grünen haben also vermehrt damit begonnen, diese chancenlosen Gesetzesinitiativen zu nutzen, um ihre eigenen Themen zu platzieren, um so Öffentlichkeit zu erzielen und ihre eigene Themenhoheit zu stärken. Zudem decken die Entwürfe nun mehr Themen ab: Sie können nach 2011 insgesamt zwölf unterschiedlichen Minor Codes (elf im ersten, fünf im zweiten Abschnitt) zugeordnet werden, während diese Anzahl 2007 bis 2008 noch bei drei lag. Diese Entwicklung kann als Indiz für eine Diversifikation dienen. In Österreich hingegen hat der Anteil der Gesetzentwürfe, die grüne Themen behandelten, von 45 auf 32 (2013 bis 2015) bzw. 36 (2016 bis 2017) Prozent abgenommen und liegt nun unterhalb des Anteils in Deutschland. Die thematische Varianz

48 KAS - Konrad-Adenauer-Stiftung, Koalitionsvertrag zwischen CDU, CSU und SPD, https:// www.kas.de/c/document_library/get_file?uuid=16f196dd-0298-d416-0acb-954d2a6a9d8d \&groupId=252038, (Abruf am 25. September 2019), S. 43. 
innerhalb des eigenen Themenspektrums hat von 14 auf insgesamt 20 Minor Codes (elf 2013 bis 2015 und 262016 bis 2017) zugenommen. Die Grünen, vor allem die deutschen, nutzen in Gesetzentwürfen also immer stärker eigene Themen, und deren Bandbreite wird größer.

\section{Grüne Gesetzentwürfe: Zwang zur Positionierung führt in Deutschland zu steigender Anzahl-zunehmende Divergenz der Themen in beiden Ländern}

Die inhaltsanalytische Auswertung von Gesetzesinitiativen erweist sich als gut geeignet, um der Frage nachzugehen, für welche Themen grüne Parteien in Deutschland und Österreich in Zeiten Großer Koalitionen das Initiativrecht nutzen. So waren bereits vor dem endgültigen Atomausstieg 2011 die deutschen Grünen 2007 bis 2008 nicht im Themenfeld der Atompolitik aktiv. Aber in Deutschland zeigt sich eine Zunahme der alleinigen Gesetzentwürfe, was darauf hindeutet, dass deren aufmerksamkeitsschaffende Funktion im zweiten Zeitraum stärker wahrgenommen wurde als im ersten. Zwischen den ersten beiden Zeiträumen hat sich zudem der Anteil der Vorlagen, die grüne Kernthemen betreffen, mehr als verdoppelt. Auch diese Entwicklung ist ein Hinweis darauf, dass Initiativen nun vermehrt zum Transport der eigenen Positionen in relevanten Themenfeldern dienen. Hier wird deutlich, dass sich eine unter Druck geratene Oppositionsfraktion stärker auf eigene Themen fokussiert; dies ist jedoch nicht in Österreich zu beobachten - hier bestand allerdings auch nicht wie in Deutschland nach 2011 der Zwang, sich wegen des Wegfalls eines zentralen Issues deutlich zu positionieren. Ein weiterer Befund betrifft die thematische Vielfalt der Entwürfe. Die festgestellte zunehmende Diversifikation in beiden Ländern weist darauf hin, wie dort Initiativen mehr und mehr dazu genutzt werden, sich ein vielfältiges grünes Profil zu schaffen.

Einschränkend ist zu bemerken, dass die Analyse nur auf zwei Phasen Großer Koalitionen beruht, die jeweils in Zwei-Jahres-Abschnitte unterteilt wurden. Daher wäre es für weiterführende Untersuchungen denkbar, weitere Zeiträume, beispielsweise darauffolgende kleinere Regierungskoalitionen einzubeziehen, um Unterschiede oder Gemeinsamkeiten in der Initiativtätigkeit auffinden zu können. Zusätzlich könnte die Berücksichtigung weiterer kleiner Oppositionsfraktionen wie FDP oder NEOS zeigen, ob sich die Ergebnisse bei anderen Parteifamilien wiederfinden lassen. Zuletzt sind natürlich weitere, geringfügig unterschiedliche Definitionen grüner Themen denkbar, die auf anderen Grundlagen wie beispielsweise Experteninterviews beruhen.

Unsere Studie zeigt, wie eine unter Druck geratene Oppositionspartei ihr parlamentarisches Themenprofil nicht nur diversifiziert, sondern das Mittel der Gesetzesinitiative verstärkt einsetzt. Dass dies kein genereller „grüner Trend“ ist, belegt der Vergleich mit Österreich; dort lässt sich diese Entwicklung nur in Teilen wiederfinden. Auch dort kam es zu einer stärkeren Diversifikation der Themen, jedoch verringerte sich der Anteil von Initiativen mit grünen Themen generell. Eine Analyse der Initiativtätigkeit von Oppositionsfraktionen lohnt sich also: Auch wenn objektiv betrachtet kaum Erfolgschancen bestehen, werden Gesetzentwürfe vermehrt eingebracht und können beispielsweise als Grundlage für innerparlamentarisches und massenmediales Agenda-Setting dienen. Wie jedoch die Umsetzung eingebrachter Vorlagen in mediale Berichterstattung erfolgt, muss in weiteren Studien geklärt werden. 